\title{
ON THE STRUCTURE OF COLLINEATION GROUPS OF FINITE PROJECTIVE PLANES
}

\author{
By WILLIAM M. KANTOR $\dagger$
}

[Received 31 December 1973-Revised 24 September 1974]

\section{Introduction}

The study of a collineation group $\Gamma$ of a finite projective plane $\mathscr{P}$ splits into two parts: the determination first of the abstract structure of $\Gamma$, and then of the manner in which $\Gamma$ acts on $\mathscr{P}$. If $\Gamma$ contains no perspectivities, almost nothing general is known about it. We shall study both questions in the case where $\Gamma$ is generated by involutory perspectivities. To avoid unstructured situations, $\Gamma$ will be assumed to contain at least two such perspectivities having different centres or axes. The determination of the structure of $\Gamma$ is then not difficult if the order $n$ of $\mathscr{P}$ is even (see $\S 8$, Remark 1). Consequently, we shall concentrate on the case of odd $n$. The main reason for the difficulties occurring for odd $n$ is that the permutation representation of $\Gamma$ on the set of centres (or axes) of involutory perspectivities has no nice group-theoretic properties; this is the exact opposite of the situation occurring for even $n$.

As usual, $Z(\Gamma)$ and $O(\Gamma)$ will denote the centre and largest normal subgroup of odd order of $\Gamma ; \sigma^{\Gamma}$ denotes the conjugacy class of $\sigma$ in $\Gamma$; and $C_{\Gamma}(\Lambda)$ and $\langle\Lambda\rangle$ are the centralizer of, and subgroup generated by, the subset $\Lambda$.

TheOREm A. Let $\mathscr{P}$ be a finite projective plane of odd order $n$, and $\Gamma$ a collineation group of $\mathscr{P}$ generated by involutory homologies and $O(\Gamma)$. Assume that $\Gamma$ contains commuting involutory homologies having different axes, and that there is no involutory homology $\sigma$ for which $\sigma O(\Gamma) \in Z(\Gamma / O(\Gamma))$. Then the following hold.

(i) There is an involutory homology $\sigma$ central in a Sylow 2-subgroup $T$ of $\Gamma$.

(ii) There exist a Klein group $\Sigma$ containing $\sigma$, and a 3-element $\varphi \in \Gamma$ normalizing $\Sigma$, such that $\langle\varphi\rangle$ acts transitively on $\Sigma \backslash\{1\}$ by conjugation.

(iii) Let $\Gamma^{*}$ be the group generated by the involutory homologies in $\Gamma$, so $\Gamma=\Gamma^{*} O(\Gamma)$. Then either (a) $O(\Gamma) \leqslant C_{\Gamma}\left(\Gamma^{*}\right)$ and $O\left(\Gamma^{*}\right) \leqslant Z\left(\Gamma^{*}\right)$, or (b) $\Sigma \triangleleft \Gamma, \Gamma$ induces $S_{3}$ on the set $\{x, y, z\}$ of centres of the involutions in $\Sigma$, $\Gamma \triangleright \Gamma_{x y z} \geqslant O(\Gamma)$, and $\Gamma_{x y z}$ centralizes $\Sigma$.

(iv) If (b) does not hold in (iii), then $C_{\Gamma}\left(\Gamma^{*}\right)$ is planar, and $\Gamma / C_{\Gamma}\left(\Gamma^{*}\right)$ acts faithfully on its fixed subplane.

† This research was supported in part by NSF Grant GP-37982X.

Proc. London Math. Soc. (3) 32 (1976) 385-402

5388.3.32 
COROLLARY. If $\Sigma$ is not a normal subgroup of $\Gamma$ and $\Gamma=\Gamma^{*}$ is generated by involutory homologies, then $O(\Gamma) \leqslant Z(\Gamma), Z(\Gamma)$ is planar, and $\Gamma / Z(\Gamma)$ acts faithfully on the fixed-point subplane of $Z(\Gamma)$ as a collineation group satisfying the hypotheses of the theorem.

Here, the hypothesis concerning involutions with different axes is not severe-see $\S 8$, Remark 2. The assumed non-existence of an involutory homology inducing a central involution of $\Gamma / O(\Gamma)$ is essential (this is seen, for example, by considering the group of all collineations of $\mathrm{PG}(2, q)$ fixing a line). The hardest of the conclusions of the theorem is that, in general, $O(\Gamma)$ centralizes all involutory homologies in $\Gamma$. Statements (i) and (ii) represent a first step towards the determination of the possible groups $\Gamma / O(\Gamma)$; statement (iv) and the corollary indicate that, for such a determination, without loss of generality $O(\Gamma)$ may be assumed to be trivial. More can be said about $\Gamma$ in this theorem (cf. $\S 7$ ), and it seems likely that all possible groups $\Gamma$ will be determined in the near future. In one case, the required group-theoretic classification theorems are available, namely, when no Baer involutions are present.

Theorem B. Let $\mathscr{P}$ and $\Gamma$ be as in Theorem A. Assume that $\Gamma$ is generated by its involutory homologies, and contains no Baer involution. Then one of the following holds.

(a) $\Gamma$ is isomorphic to $\operatorname{PSL}(2, q), \operatorname{PGL}(2, q), \operatorname{PSL} \wedge(2,9), \operatorname{PSL}(3, q)$, $\mathrm{SL}(3, q), \mathrm{PSU}(3, q), \mathrm{SU}(3, q), \mathrm{A}_{7}, \hat{\mathrm{A}}_{7}$, or $\mathrm{PSU}(3,4)$; here, $q$ is an odd prime power.

(b) $\Gamma$ has a 2-subgroup $\left\langle\varphi_{1}\right\rangle \times\left\langle\varphi_{2}\right\rangle$ with $\left|\varphi_{1}\right|=\left|\varphi_{2}\right| \geqslant 2$, such that $\Gamma \triangleright O(\Gamma)\left\langle\varphi_{1}, \varphi_{2}\right\rangle$ and $\Gamma / O(\Gamma)\left\langle\varphi_{1}, \varphi_{2}\right\rangle \approx \mathrm{S}_{3}$; moreover $\Gamma$ induces $\mathrm{S}_{3}$ on the set $\{x, y, z\}$ of centres of the involutions in the Klein group $\Sigma$ of $\left\langle\varphi_{1}, \varphi_{2}\right\rangle$, and $\Gamma_{x y \varepsilon}=O(\Gamma)\left\langle\varphi_{1}, \varphi_{2}\right\rangle \geqslant O(\Gamma) \times \Sigma$.

(Here, $\operatorname{PSL}^{\wedge}(2,9)$ and $\hat{A}_{7}$ are non-split extensions of $\operatorname{PSL}(2,9)$ and $A_{7}$ by groups of order 3.) Thus the structure of $\Gamma$ is completely known in this setting, and, in particular, when $n$ is not a square. Which of the above groups can actually occur for a given $n$ is, however, not known. Note that $\operatorname{PSU}(3,5)$ has a subgroup isomorphic to $A_{7}$; on the other hand $\operatorname{PSU}(3,4)$ probably cannot occur.

Theorem C. Let $\mathscr{P}$ and $\Gamma$ be as in Theorems $A$ and $B$.

(i) If $\Gamma$ is isomorphic to $\operatorname{PSL}(3, q)$ or $\mathrm{SL}(3, q)$, then $\mathscr{P}$ has a $\Gamma$-invariant desarguesian subplane of order $q$ on which $\Gamma$ induces $\operatorname{PSL}(3, q)$, and all elations of the subplane are induced by elations of $\mathscr{P}$. Moreover, $q \mid n$, $(q-1) \mid(n-1)$, and $(q+1) \mid\left(n^{2}-1\right)$. 
(ii) If $\Gamma$ is isomorphic to $\mathrm{PGL}(2, q)$ with $(q, n)>1$ and $q>3$, then $\mathscr{P}$ has $a \Gamma$-invariant desarguesian subplane of order $q$ having an orthogonal polarity preserved by $\Gamma$. Moreover, $q|n,(q-1)|(n-1)$, and $(q+1) \mid\left(n^{2}-1\right)$.

(iii) If $\Gamma$ is isomorphic to $\mathrm{PSU}(3, q)$ or $\mathrm{SU}(3, q)$ with $(q, n)>1$, then $\mathscr{P}$ has a desarguesian $\Gamma$-invariant subplane of order $q^{2}$ having a unitary polarity preserved by $\Gamma$. Moreover, $q\left|n^{2},(q-1)\right|(n-1)$, and $(q+1) \mid\left(n^{2}-1\right)$.

Only fragmentary results are known for the remaining cases of Theorem $\mathrm{B}(\mathrm{a})$, or when $(q, n)=1$ (see $\S 8$, Remark 3). In particular, the case in which $\Gamma \approx \operatorname{PSL}(2, q)$ with $q \equiv 3 \bmod 4$ seems especially difficult: none of our techniques provide information here.

Weaker versions of parts of Theorems A, B, and C were described in [19]. A special case of Theorem B first appeared in [17, (2.5) ]. (However, none of the main results of [17 and 18] are consequences of the results of the present paper.) Special cases of Theorem $\mathrm{C}$ were proved in $[\mathbf{6}, \mathbf{1 6}, \mathbf{2 1}$, 24, 27, and 28]. No part of Theorem $\mathrm{C}$ is known in general without the assumption that involutions are perspectivities; however, if $n$ is equal to $q$ or $q^{2}$, results exist asserting that $\mathscr{P}$ is a desarguesian or Hughes planesee the aforementioned references. Results such as Theorem $\mathrm{C}$ are discussed for even $n$ in $\S 8$, Remark 1.

The organisation of the paper is as follows. In $\S 2$ some known grouptheoretic facts are stated for reference. Section 3 contains known or elementary geometric facts. In $\S 4$ we prove a result concerning a very special type of solvable collineation group, from which Theorem A(iii) follows easily. In $\S 5$ Theorems A and B are proved, and in $\S 6$ Theorem $C$ is. Variations and extensions of our results are discussed in $\S 8$. In particular, infinite versions of Theorems A and B are discussed in $\S 8$, Remark 5.

I am indebted to the referee for numerous helpful suggestions.

\section{Group-theoretic preliminaries}

If $G$ is a group, then $Z(G)$ is its centre, $G^{\prime}$ is its commutator subgroup, and $O(G)$ is its largest normal subgroup of odd order. If $X$ is a subset of $G$, then $N_{G}(X)$ and $C_{G}(X)$ are its normalizer and centralizer. $x^{y}=x^{-1} y x$, $[x, y]=x^{-1} y^{-1} x y$, and if $X$ and $Y$ are non-empty subsets of $G,\langle X\rangle$ is the subgroup generated by $X, X^{a}$ is the set of conjugates of $X$, and $[X, Y]=\langle[x, y] \mid x \in X, y \in Y\rangle$.

(2.1) Let $A, B \leqslant G$ with $B \leqslant N_{G}(A), A$ elementary abelian, and $(|A|,|B|)=1$. Then $A=C_{A}(B) \times[A, B]$, where both factors are normalized by $N_{G}(A) \cap N_{G}(B)$. 
Proof. See [12, p. 177].

(2.2) Let $X \unlhd Y \leqslant G$. Let $A \leqslant B$ be a p-group centralizing $X$, normalizing $Y$, and centralizing $Y / X$. If $p \nmid|Y|$, then $A$ centralizes $Y$.

Proof. See [12, p. 224].

(2.3) (Glauberman's $Z^{*}$-theorem.) Suppose $G>O(G)$ and

$$
Z(G / O(G))=1 \text {. }
$$

Then, for each involution $t \in G$ and each Sylow 2-group $S$ of $G$ containing $t$, $C_{S}(t) \backslash\{t\}$ contains a conjugate of $t$.

Proof. See [10].

(2.4) (Burnside's fusion lemma.) Let $P$ be a Sylow p-subgroup of $G$, and let $X$ and $Y$ be non-empty unions of conjugacy classes of $P$. If $X$ and $Y$ are conjugate in $G$, then they are conjugate in $N_{G}(P)$.

Proof. See [5, p. 155].

(2.5) Let $P$ be a Sylow p-subgroup of $G, z \in Z(P)$ with $|z|=p$, and $g \in P \backslash Z(P)\left(g \in z^{\alpha}\right)$. Then there is an elementary abelian p-group $E$ containing $z$ such that $N_{G}(E)$ has an element $x$ for which $z^{x} \neq z$ and $p \nmid|x|$.

Proof. See [5, p. 156].

(2.6) (Thompson's transfer lemma.) Let $S$ be a Sylow 2-subgroup of $G$, $U<S$, and $S=\langle t\rangle U$ for an involution $t$. Assume that $t$ is conjugate in $G$ to no element of $U$. Then there exists $H<G$ with $G=H\langle t\rangle$.

Proof. See [12, p. 265, Ex. 3(i)].

(2.7) Let $G$ be a group of even order, generated by its involutions, such that $O(G)=Z(G)=1$. If $G$ has no elementary abelian subgroup of order 8 , then one of the following holds:

(i) $G$ is isomorphic to $\operatorname{PSL}(2, q), \operatorname{PGL}(2, q), \operatorname{PSL}(3, q), \operatorname{PSU}(3, q), \mathrm{A}_{7}, \mathrm{M}_{11}$, or $\mathrm{PSU}(3,4)$, where $q$ is an odd prime power;

(ii) $G \triangleright C_{1} \times C_{2}$, with $C_{1}$ and $C_{2}$ cyclic, $\left|C_{1}\right|=\left|C_{2}\right| \geqslant 2$, and

$$
G /\left(C_{1} \times C_{2}\right) \approx \mathrm{S}_{3} \text {. }
$$

Proof. Let $H$ be a minimal normal subgroup of $G$. Then $H$ is an elementary abelian 2-group or a direct product of non-abelian simple groups.

In the latter case, by $[1], H$ is isomorphic to $\operatorname{PSL}(2, q), \operatorname{PSL}(3, q)$, $\operatorname{PSU}(3, q), \mathrm{M}_{11}, \mathrm{~A}_{7}$, or $\operatorname{PSU}(3,4)$ for some odd $q$. Since $C_{G}(H)$ contains no involutions and is normal in $G, C_{G}(H) \leqslant O(G)=1$. Thus $G \leqslant$ Aut $H$. But 
$G$ is generated by its involutions. Hence (i) holds as $G$ has no elementary abelian subgroup of order 8.

Thus we may suppose that $H$ is a Klein group. Clearly, $O\left(C_{G}(H)\right)=1$ and $\left|G: C_{G}(H)\right|=3$ or 6 . In particular, if $X=\langle g \in G| g$ has odd order $\rangle$, then $H \leqslant X^{\prime}$ and $\left|X: C_{X}(H)\right|=3$. Clearly, $O(X)=1$ and $X$ has no subgroup of index 2 . Since $H<X$, we also have $Z(X)=1$.

Since $\left|X: C_{X}(H)\right|=3, C_{X}(H)$ contains a Sylow 2-subgroup $S$ of $X$. By the remark at the end of [1] (compare [3]), $S=C_{1} \times C_{2}$ with $C_{1}$ and $C_{2}$ cyclic of the same order. By the Frattini argument, $\left|N_{X}(S): C_{X}(S)\right|=3$.

We now use induction to show that $S$ is normal in $X$ of index 3. This is obvious if $S=H$, so suppose $S>H$. Then $S / H$ is Sylow in $X / H$. Also $X / H$ has no subgroup of index 2 and no central involution, and $O(X / H)=1$. For, let $Y / H=O(X / H)$. By the Frattini argument, $X=H N_{X}(O(Y))$. Since $S>H, N_{X}(O(Y))$ contains a Klein group of $X$, which must be $H$. Thus $[O(Y), H] \leqslant O(Y) \cap H=1$, and hence

$$
O(Y) \leqslant O(X)=1 \text {, }
$$

so $Y=H$. Consequently $O(X / H)=1$.

There are now two possibilities: (a) $X / H$ has a normal Klein subgroup $K / H$; or (b) $X / H$ has a normal subgroup $K / H \approx \operatorname{PSL}(2, q)$ for some odd $q>3$ and $|S / H|=4$.

Assume (a) holds. Then, by induction, $S / H$ is normal in $X / H$ of index 3. Then $S$ is normal in $X$ of index 3. Since $O\left(C_{G}(H)\right)=1$, it follows that (ii) holds here.

Now assume (b) holds. We know that $H \leqslant X^{\prime}$. Also, since $K / H$ is simple, $H \leqslant Z(K)$. However, no such central extension of $\operatorname{PSL}(2, q)$ exists, by [26]. Thus (b) cannot occur.

(2.8) Let $L$ be a group such that $|Z(L)|$ is odd and $L / Z(L)$ is one of the non-solvable groups in (2.7)(i). If $L$ is generated by its involutions, then $L$ is isomorphic to $\operatorname{PSL}(2, q), \operatorname{PGL}(2, q), \operatorname{PSL}(3, q), \operatorname{SL}(3, q), \operatorname{PSU}(3, q)$, $\mathrm{SU}(3, q), \mathrm{A}_{7}, \mathrm{M}_{11}, \mathrm{PSU}(3,4)$, or unique (up to isomorphism) groups $\operatorname{PSL}(2,9)$ and $\hat{\mathrm{A}}_{7}$ having centres of order 3 with quotient groups $\operatorname{PSL}(2,9)$ and $\mathrm{A}_{7}$.

Proof. Let $H$ be the last term of the commutator series of $L$. Clearly $|Z(H)|$ is odd and $H / Z(H)$ is one of the groups in (2.7)(i) other than $\operatorname{PGL}(2, q)$. Then $H$ is isomorphic to $\operatorname{PSL}(2, q), \operatorname{PSL}(2,9), \operatorname{PSL}(3, q)$, $\mathrm{SL}(3, q), \operatorname{PSU}(3, q), \mathrm{SU}(3, q), \mathrm{A}_{7}, \hat{\mathrm{A}}_{7}, \mathrm{M}_{11}$, or $\operatorname{PSU}(3,4)$ (see [13]). If $L / Z(L)$ is not isomorphic to $\operatorname{PGL}(2, q)$, all its involutions are conjugate. Since each coset of $Z(L)$ in $L$ has at most one involution, $L$ has a single class of involutions, all of which are in $H$. Thus $H=L$ here. 
If $L / Z(L) \approx \mathrm{PGL}(2, q)$, there is an involution $t \in L \backslash H$ such that $H\langle t\rangle / Z(H) \approx \mathrm{PGL}(2, q)$ and $L=(H\langle t\rangle) Z(L)$. Thus $H\langle t\rangle$ is normal in $L$, hence it contains all involutions in $L$, and thus $H\langle t\rangle$ is $L$.

It remains only to show that we cannot have $L=H\langle t\rangle$ and $H=\operatorname{PSL}^{\wedge}(2,9)$. Suppose this can occur. A Sylow 3-subgroup $T$ of $L$ is extraspecial of order 27. By the Frattini argument, $N_{L}(T)$ has an element of order 8 , centralizing $Z(T)=Z(L)$, and faithful on $T / Z(T)$. However, an extraspecial group of order 27 has no such automorphism.

\section{Geometric preliminaries}

Let $\mathscr{P}$ be a finite projective plane of order $n$. It will be convenient to write $p p=p$ and $L \cap L=L$ for each point $p$ and line $L$. If $\sigma$ is a nontrivial perspectivity, $c_{\sigma}$ and $A_{\sigma}$ will denote its centre and axis. If $\Lambda$ is any set of collineations of $\mathscr{P}$ fixing a quadrangle pointwise, $\mathscr{P}_{\Lambda}$ denotes its fixed point subplane. If $\Gamma$ is any collineation group and $S$ any set consisting of more than one point, $\Gamma(S)$ is the pointwise stabilizer of $S$. $\Gamma(L)$ and $\Gamma(x)$ are the groups of perspectivities with axis $L$ and centre $x$, and $\Gamma(x, L)=\Gamma(x) \cap \Gamma(L)$.

Throughout this section, we shall assume $n$ is odd.

(3.1) Let $\sigma$ and $\tau$ be commuting involutory homologies having different axes. Then

(i) $\sigma \tau$ is an involutory $\left(A_{\sigma} \cap A_{\tau}, c_{\sigma} c_{\tau}\right)$-homology,

(ii) $\sigma$ is the only involutory $\left(c_{\sigma}, A_{\sigma}\right)$-homology, and

(iii) any collineation fixing $c_{\sigma}$ and $A_{\sigma}$ centralizes $\sigma$.

Proof. See [23 and 20] (or [8, p. 120]) for proofs of (i) and (ii). Clearly (ii) implies (iii).

(3.2) Let $\Gamma$ be a collineation group of $\mathscr{P}$ containing $\sigma$ and $\tau$ as in (3.1). Then

(i) if $\sigma^{\prime}$ and $\tau^{\prime}$ are distinct commuting involutory homologies in $\Gamma$, $A_{\sigma^{\prime}} \neq A_{\tau^{\prime}} ;$ and

(ii) $\Gamma$ contains no elementary abelian subgroup of order 8 generated by three homologies.

Proof. (i) Suppose $A_{\sigma^{\prime}}=A_{\tau^{\prime}}$. Then $\sigma$ and $\sigma^{\prime}$ are not conjugate, so $\left|\left\langle\sigma, \sigma^{\prime}\right\rangle\right|$ must be divisible by 4. Let $\left\langle\sigma, \sigma^{\prime \prime}\right\rangle$ be a Sylow 2-subgroup of $\left\langle\sigma, \sigma^{\prime}\right\rangle$, with $\sigma^{\prime \prime}$ conjugate to $\sigma^{\prime}$. Let $\rho$ be the involution in $\left\langle\sigma \sigma^{\prime \prime}\right\rangle$. Then $\rho$ is the product of involutory homologies, and hence is one by (3.1)(i). $\rho$ commutes with $\sigma$ and $\sigma^{\prime \prime}$. By (3.1)(ii) applied to $\sigma$ and $\tau, A_{\sigma} \neq A_{\rho}$ so (3.1)(ii) applies to $\sigma$ and $\rho$. Then (3.1)(ii) must also apply to $\rho$ and $\sigma^{\prime \prime}$. 
But $\sigma^{\prime}$ is conjugate to $\sigma^{\prime \prime}$, so (3.1)(ii) must apply to $\sigma^{\prime}$ and $\tau^{\prime}$, which is not the case.

(ii) See [17, (2.2)] for a proof of (ii).

(3.3) Proposition. Let $\Gamma$ be a collineation group of $\mathscr{P}$, where $\mathscr{P}$ has odd order $n$. If $\Gamma$ contains an involutory homology, then so does the centre of a Sylow 2-subgroup of $\Gamma$.

Proof. Deny this, and let $\sigma$ be an involutory homology such that a Sylow 2-subgroup $\Sigma$ of $C_{\Gamma}(\sigma)$ has the largest possible order. Since $\Sigma$ is not Sylow in $\Gamma, \Sigma<\Pi \leqslant \Gamma$ with $|\Pi: \Sigma|=2$. If $\pi \in \Pi \backslash \Sigma$, then $\pi$ interchanges $\sigma$ and $\sigma^{\prime}=\sigma^{\pi}$, and hence normalizes $\left\langle\sigma, \sigma^{\prime}\right\rangle$. Then $\Pi$ centralizes $\tau=\sigma \sigma^{\prime}$. The maximality of $\Sigma$ shows that $\tau$ cannot be a homology. However, if $A_{\sigma}=A_{\sigma^{\prime}}$, then $\tau$ is certainly a homology, while if $A_{\sigma} \neq A_{\sigma^{\prime}}$, then $\tau$ is a homology by (3.1).

(3.4) Let $\sigma$ and $\tau$ be distinct involutory homologies such that $|\sigma \tau|$ is $a$ power of a prime $p$.

(i) Each fixed point of $\sigma \tau$ is in $A_{\sigma} \cap A_{\tau}$ or in $c_{\sigma} c_{\tau}$, unless $c_{\sigma}=c_{\tau}$ and $\sigma \tau$ is a $\left(c_{\sigma}, c_{\sigma}\left(A_{\sigma} \cap A_{\tau}\right)\right)$-elation.

(ii) $|\sigma \tau|$ divides $n\left(n^{2}-1\right)$.

(iii) If $c_{\sigma}=c_{\tau}$, then either $A_{\sigma}=A_{\tau}$ and $p \mid(n-1)$, or else $A_{\sigma} \neq A_{\tau}, p \mid n$, and $\sigma \tau$ is a $\left(c_{\sigma}, c_{\sigma}\left(A_{\sigma} \cap A_{\tau}\right)\right)$-elation.

(iv) If $c_{\sigma} \neq c_{\tau}, A_{\sigma} \neq A_{\tau}$, and $A_{\sigma} \cap A_{\tau} \notin c_{\sigma} c_{\tau}$, then $\mid \sigma \tau \|(n-1)$ or $|\sigma \tau| \mid(n+1)$.

Proof. Anything proved for $\sigma \tau$ will clearly hold for each of its powers. Suppose $\sigma \tau$ fixes a point $x$, where $x$ is in neither $c_{\sigma} c_{\tau}$ nor $A_{\sigma} \cap A_{\tau}$. Then $c_{\sigma}, x, x^{\sigma}$ and $c_{\tau}, x, x^{\tau}$ are collinear triples. Since $x^{\sigma}=x^{\tau}$, it follows that $c_{\sigma}, c_{\tau}, x$ are collinear. Since $x \notin c_{\sigma} c_{\tau}$, we must have $c_{\sigma}=c_{\tau}$, so $\sigma \tau$ has centre $c_{\sigma}$. Thus either $A_{\sigma}=A_{\tau}$, in which case $x \notin A_{\sigma} \cap A_{\tau}$ is impossible, or $A_{\sigma} \neq A_{\tau}$ and $\sigma \tau$ is a $\left(c_{\sigma}, c_{\sigma}\left(A_{\sigma} \cap A_{\tau}\right)\right)$-elation (see [8, p. 120]). Moreover, $p \mid n$ here.

Suppose $c_{\sigma} \neq c_{\tau}$ and $A_{\sigma} \neq A_{\tau}$. If $A_{\sigma} \cap A_{\tau} \in c_{\sigma} c_{\tau}$, then $\sigma \tau$ fixes no point off $c_{\sigma} c_{\tau}$, so $p \mid n^{2}$. If $A_{\sigma} \cap A_{\tau} \notin c_{\sigma} c_{\tau}$ and $\sigma \tau$ fixes some $x \in c_{\sigma} c_{\tau}$, then $\sigma \tau$ fixes no point of $\left(A_{\sigma} \cap A_{\tau}\right) x \backslash\left\{A_{\sigma} \cap A_{\tau}, x\right\}$ and $\mid \sigma \tau \|(n-1)$ (so $\left.p \mid(n-1)\right)$; if, however, $\sigma \tau$ fixes no point of $c_{\sigma} c_{\tau}$, then $p \mid(n+1)$, so $\langle\sigma \tau\rangle$ is semiregular on $c_{\sigma} c_{\tau}$ and $\mid \sigma \tau \|(n+1)$.

If $A_{\sigma}=A_{\tau}$ and $c_{\sigma}=c_{\tau}$, then $\sigma \tau$ is clearly a $\left(c_{\sigma}, A_{\sigma}\right)$-homology, so $|\sigma \tau| \mid(n-1)$.

It is not difficult to see that (3.1), (3.2), (3.3), and (3.4)(i) also hold for finite collineation groups of infinite projective planes. The same is true 
of the next result, although slightly more care is needed (and, of course, the assertion that $3 \mid(n-1)$ must be deleted in (3.5)(iii)).

(3.5) TheOREM. Let $p$ be an odd prime, and $\Pi$ a non-trivial $p$-subgroup of a collineation group $\Gamma$. Suppose $\Pi$ is inverted by an involutory homology $\sigma \in \Gamma$. Then precisely one of the following holds:

- (i) all centres of involutions of $\Pi\langle\sigma\rangle$ coincide and $N_{\Gamma}(\Pi)$ fixes $c_{\sigma}$, or dually;

(ii) there are two involutions of $\Pi\langle\sigma\rangle$ having different centres and axes, all centres of involutions in $\Pi\langle\sigma\rangle$ lie on a line fixed by $N_{\Gamma}(\Pi)$, and all axes of involutions in $\Pi\langle\sigma\rangle$ lie on a point fixed by $N_{\Gamma}(\Pi)$;

(iii) $p=3$ divides $n-1$, and each $\pi \in \Pi \backslash\{1\}$ fixes exactly three points which are non-collinear and are permuted transitively by $\Pi$;

(iv) $\Pi$ fixes exactly three points $x, y, z$, which are non-collinear, $x^{\sigma}=y$, $z^{\sigma}=z$, and $N_{\Gamma}(\Pi)$ induces $\mathrm{S}_{3}$ on $\{x, y, z\}$.

Proof. Since $\Pi$ is abelian, $\Pi$ acts on the sets of fixed points and lines of each of its elements. Let $\pi \in \Pi \backslash\{1\}$. Then $\tau=\sigma \pi$ is an involution conjugate to $\sigma$, and $\pi=\sigma \tau$.

Case 1. $A_{\sigma} \neq A_{\tau}$ and $c_{\sigma} \neq c_{\tau}$. By (3.4), the set of fixed points of $\pi$ consists of $x=A_{\sigma} \cap A_{\tau}$ and some points on $L=c_{\sigma} c_{\tau}$; we do not know whether $x \in L$ or $x \notin L$. Let $\pi$ and $\Pi$ fix $f_{\pi}$ and $f_{\Pi}$ points of $L$, respectively.

We first claim that, if $N_{\Gamma}$ (П) fixes $x$, then (ii) holds. For, since $x \in A_{\sigma}$ and all involutions in $\Pi\langle\sigma\rangle$ are conjugate, the axes of all these involutions pass through $x$. Thus if $N_{\Gamma}(\Pi)$ also fixes $L$, then (ii) holds. Suppose $\varphi \in N_{\Gamma}(\Pi)$ moves $L$. Then either $c_{\sigma}{ }^{\phi}$ or $c_{\tau}{ }^{\phi}$ is not on $L$. Suppose, say, $c_{\sigma}{ }^{\Phi} \notin L$, and write $\rho=\sigma^{\Phi}$. Then $c_{\sigma} \neq c_{\rho} \notin L$ and $c_{\rho} \neq x \in A_{\rho}$. Consider $\sigma \rho$. Since $\sigma$ and $\rho$ invert $\Pi, \sigma \rho$ centralizes $\Pi$. Also, $\sigma \rho$ fixes $x$ and $M=c_{\sigma} c_{\rho}$. Note that $\Pi$ moves $M$ (as otherwise $\Pi$ would fix $c_{\sigma}=L \cap M$, and then $\tau \in \sigma^{\Pi}$ would yield $\left.c_{\sigma}=c_{\tau}\right)$. On the other hand, $C_{\Gamma}(\sigma \rho) \geqslant \Pi$ permutes the fixed points and lines of $\sigma \rho$. Let $f_{\sigma \rho}$ denote the number of fixed points of $\sigma \rho$ on $M$. If $x \in M$, then $x \notin c_{\rho} c_{\tau}$ and $\sigma \rho$ centralizes $\rho \tau=(\sigma \rho)^{-1} \sigma \tau$, so $\sigma \rho$ fixes $c_{\rho} c_{\tau} \cap M \neq x, f_{\sigma \rho} \geqslant 2$, and $M$ is the unique fixed line of $\sigma \rho$ having at least two fixed points. Thus $M^{\Pi} \neq M$ implies that $x \notin M$. Certainly $x$ is the only fixed point of $\sigma \rho$ off $M$. If $f_{\sigma \rho}=0$, then $M$ is the unique fixed line of $\sigma \rho$ having no fixed points. If $f_{\sigma \rho}=1$, then $M$ is the unique fixed line of $\sigma \rho$ having just one point (since all other fixed lines must pass through $x$ ). If $f_{\sigma \rho} \geqslant 3$, then $M$ is the unique fixed line of $\sigma \rho$ having more than two fixed points. Since $\Pi$ must move $M$, it follows that $f_{\sigma \rho}=2$. But $\Pi$ fixes $x$, and hence permutes the pair $\{y, z\}$ of fixed points $(\neq x)$ of $\sigma \rho$, so $\Pi$ fixes $y z=M$. This contradiction proves our claim.

Dually, if $N_{\Gamma}(\Pi)$ fixes $L$, then (ii) holds. 
We now consider the various possibilities for $f_{\pi}$ and $f_{\mathrm{n}}$.

If $f_{\pi}=0$, then $L$ is the unique fixed line of $\pi$, and hence of $\Pi$, so $N_{\Gamma}$ (II) fixes $L$. Thus (ii) holds.

If $f_{\pi}=1$ and $x \notin L$, then $L$ is the unique fixed line of $\pi$ (and $\Pi$ ) having one fixed point (since all fixed lines not equal to $L$ pass through $x$, by (3.4)). Thus $N_{\Gamma}(\Pi)$ again fixes $L$, so (ii) holds.

Suppose $f_{\pi}=1$ and $x \in L$. Then $\pi$ fixes just one point, so $N_{\Gamma}(\Pi)$ fixes $x$ and (ii) holds.

Suppose $f_{\pi}=2$ and $x \in L$. Since $|\Pi|$ is odd, $\Pi$ must fix both fixed points of $\pi$. Thus $N_{\Gamma}(\Pi)$ fixes $L$.

Suppose $f_{\pi} \geqslant 3$. Then clearly $\Pi$ fixes $L$. If also $f_{\Pi}=0$, or $f_{\Pi}=1$ and $x \notin L$, or $f_{\Pi} \geqslant 3$ and $x \in L$, then as before, $N_{\Gamma}(\Pi)$ fixes $L$. Also, if $f_{\Pi}=1$ and $x \in L$, then $N_{\Gamma}(\Pi)$ fixes $x$. Thus we may assume that $f_{\Pi}=2$ and $x \notin L$. Then $\Pi$ fixes exactly three points. The two fixed points in $L$ are fixed by $\pi$, and hence are not fixed by $\sigma$. Thus either $N_{\Gamma}(\Pi)$ fixes $L$, and (ii) holds, or (iv) holds.

Finally, suppose $f_{\pi}=2$ and $x \notin L$. Then $\pi$ fixes exactly three points $x, y, z$, where $L=y z, x^{\sigma}=x$, and $y^{\sigma}=z$. Suppose $\Pi$ fixes $x$. Then $\Pi$ fixes exactly three points, and as before (ii) or (iv) holds.

Thus if (ii) and (iv) do not hold, then each $\pi \in \Pi \backslash\{1\}$ must fix exactly three points. Moreover, $\Pi$ acts non-trivially on these points. That is, (iii) holds.

Case 2. $A_{\sigma}=A_{\tau}$ or $c_{\sigma}=c_{\tau}$. Suppose $c_{\sigma}=c_{\tau}$, so $\pi=\sigma \tau \in \Gamma\left(c_{\sigma}\right)$. Since $\Pi$ is abelian, it fixes $c=c_{\sigma}$.

Take any involution $\rho \in \Pi\langle\sigma\rangle \backslash\langle\sigma, \tau\rangle$. We claim that $c_{\rho}=c$. For suppose $c_{\rho} \neq c$. If also $A_{\rho} \neq A_{\sigma}$ or $A_{\rho} \neq A_{\tau}$, we are back in Case 1. Thus $A_{\sigma}=A_{\rho}=A_{\tau}$. Now $\sigma \tau \in \Gamma\left(c_{\sigma}, A_{\sigma}\right)$, and $\sigma \rho \in \Pi$ commutes with $\sigma \tau \in \Pi$. Thus $\sigma \rho$ fixes $c_{\sigma}$, which is absurd.

Consequently $\Pi\langle\sigma\rangle \leqslant \Gamma(c)$, so $N_{\Gamma}(\Pi)$ fixes $c$. Thus (i) holds.

(3.6) Suppose $\Delta_{1} \times \Delta_{2}$ is a collineation group, where $\Delta_{1}$ and $\Delta_{2}$ are dihedral groups of order $2 p$ with $p$ an odd prime. Assume that all involutions in $\Delta_{1}$ and $\Delta_{2}$ are homologies. Then all elements of $\Delta_{1}$ are perspectivities having the same centre or axis.

Proof. Deny this, and write $\Delta_{1}=\langle\sigma, \tau\rangle$ and $\Delta_{2}=\langle\mu, \nu\rangle$ with $\sigma, \tau, \mu$, and $\nu$ involutory homologies. Clearly $\mu$ fixes $c_{\sigma}, c_{\tau}, A_{\sigma}, A_{\tau}, c_{\sigma} c_{\tau} \cap A_{\sigma}$, and $c_{\sigma} c_{\tau} \cap A_{\tau}$.

Suppose $A_{\sigma} \cap A_{\tau} \notin c_{\sigma} c_{\tau}$. Then we must have $c_{\mu}=A_{\sigma} \cap A_{\tau}$ and $A_{\mu}=c_{\sigma} c_{\tau}$. Similarly, $c_{\nu}=c_{\mu}$ and $A_{\mu}=A_{\nu}$. However, $\sigma \mu=\mu \sigma$ and $A_{\mu} \neq A_{\sigma}$, so that $\mu=\nu$ by (3.1)(ii). 
Thus $A_{\sigma} \cap A_{\tau} \in c_{\sigma} c_{\tau}$, so $\mu$ fixes the concurrent lines $A_{\sigma}, A_{\tau}, c_{\sigma} c_{\tau}$ and the collinear points $c_{\sigma}, c_{\tau}, A_{\sigma} \cap A_{\tau}$. This is impossible.

\section{Some solvable collineation groups}

The proof of Theorem A requires Theorem 4.1, which concerns very specific types of solvable collineation groups.

(4.1) Theorem. Let $\mathscr{P}$ be a projective plane of odd order, and let $\Delta$ be a collineation group of $\mathscr{P}$. Assume that $\Delta / O(\Delta) \approx \mathrm{A}_{4}$, and that a Sylow 2-subgroup $\Sigma$ of $\Delta$ contains involutory homologies having different axes. Then $\Sigma$ centralizes $O(\Delta)$.

Proof. Let $\mathscr{P}$ and $\Delta$ produce a counter-example to (4.1) with minimal $|\Delta|$. By the Frattini argument, $\Delta=N_{\Delta}(\Sigma) O(\Delta)$. By the Feit-Thompson theorem [9], $O(\Delta)$ is solvable. Let $\Lambda$ be a minimal normal subgroup of $\Delta$ contained in $O(\Delta)$. Then $\Lambda$ is an elementary abelian $p$-group for some prime $p \neq 2$.

Suppose that $\Sigma$ centralizes $\Lambda$. Then $\Lambda$ fixes the centres $a, b, c$ of the three involutions in $\Sigma$. Assume first that these are the only fixed points of $\Lambda$. By (3.1), $\Sigma$ centralizes $\Delta_{a b c}$. A 3-element in $N_{\Delta}(\Sigma)$ not in $O(\Gamma)$ must move these points (as $\Delta=N_{\Delta}(\Sigma) O(\Delta)$ ). Thus $\Delta_{a b c}=\Sigma \times O\left(\Delta_{a b c}\right)$ has index 3 in $\Delta$, so that $O\left(\Delta_{a b c}\right)=O(\Delta)$. However, we are assuming that $(4.1)$ is false.

Thus if $\Sigma$ centralizes $\Lambda$, then $\Lambda$ fixes a point not fixed by $\Sigma$. Since $N_{\Delta}(\Sigma)$ is transitive on the fixed points of $\Sigma$, even if this point is on a side of the fixed triangle of $\Sigma, \Lambda$ is planar. $\Delta$ induces a collineation group $\bar{\Delta}$ of $\mathscr{P}_{\Lambda}$, having a Sylow 2-subgroup $\bar{\Sigma}$ (the image of $\Sigma$ ) containing involutory homologies with different axes. Moreover, $N_{\Delta}(\Sigma)$ is transitive on these axes, so $\Delta / O(\bar{\Delta}) \approx \mathrm{A}_{4}$. By the minimality of $|\Delta|, \bar{\Sigma}$ centralizes $O(\bar{\Delta})$. Clearly, $O(\bar{\Delta}) \geqslant O(\Delta) . \Delta\left(\mathscr{P}_{\Lambda}\right) / \Delta\left(\mathscr{P}_{\Lambda}\right)$, so $\Sigma$ centralizes $O(\Delta) / O(\Delta) \cap \Delta(\mathscr{P})$. By (3.1), $\Sigma$ centralizes $\Delta\left(\mathscr{P}_{\Lambda}\right)$, and hence, by (2.2), also centralizes $O(\Delta)$.

Consequently, $\Sigma$ cannot centralize $\Lambda$. Write $\Sigma=\langle\sigma, \tau\rangle$. Since $N_{\Delta}(\Sigma)$ acts transitively on $\Sigma \backslash\{1\},\left|C_{\Lambda}(\sigma)\right|=\left|C_{\Lambda}(\tau)\right|=\left|C_{\Lambda}(\sigma \tau)\right| \neq|\Lambda|$. Moreover, since $\Lambda$ is abelian $\Lambda=C_{\Lambda}(\sigma) C_{\Lambda}(\tau) C_{\Lambda}(\sigma \tau)$.

By (3.1), $\Lambda$ does not fix any of the points $c_{\sigma}, c_{\tau}, c_{\sigma \tau}$, and hence neither can $C_{\Lambda}(\sigma)$. On the other hand, $C_{\Lambda}(\sigma)$ fixes $A_{\sigma}$ and each point of $\left(c_{\sigma}\right)^{\Lambda}$ (as $\Lambda$ is abelian). Here, $\left(c_{\sigma}\right)^{\Lambda}$ contains both $\left(c_{\sigma}\right)^{C_{\Lambda}(\tau)} \subseteq A_{\tau}$ and $\left(c_{\sigma}\right)^{c_{\Lambda(\sigma \tau)}} \subseteq A_{\sigma r}$. Since the lines $A_{\tau}, A_{\sigma \tau}$ cannot both be fixed by $C_{\Lambda}(\sigma)$, we may assume that $C_{\Lambda}(\tau)$ fixes $c_{\sigma}$. However $\Lambda=C_{\Lambda}(\sigma) C_{\Lambda}(\tau) C_{\Lambda}(\sigma \tau)$ now implies that $\left(c_{\sigma}\right)^{\Lambda} \subseteq A_{\sigma \tau}$. Thus $\Lambda$ fixes either $c_{\sigma}$ or $A_{\sigma \tau}$, which is not the case. (I am grateful to R. Liebler for providing the simplifications of my original proof contained in the present paragraph.) 
The preceding proof shows that (4.1) also holds for finite collineation groups of infinite planes.

\section{Theorems $\mathbf{A}$ and $\mathbf{B}$}

Proof of Theorem A. (i) See (3.3) for this result.

(ii) By (2.3), there is a conjugate $\tau$ of $\sigma$ such that $\langle\sigma, \tau\rangle$ is a Klein group. Let $T$ be a Sylow 2-subgroup of $\Gamma$ containing $\langle\sigma, \tau\rangle$ with $\sigma \in Z(T)$.

If $\langle\sigma, \tau\rangle \leqslant Z(T)$, then (2.4) shows that $N(T)$ moves $\sigma$ to $\tau$. In this case $N(T)$ must normalize $\langle\sigma, \tau\rangle$ by (3.2)(ii). Since $N(T)$ must induce a group of odd order on $\langle\sigma, \tau\rangle$ (as $T$ is Sylow in $\Gamma$ ), (ii) follows.

If $\langle\sigma, \tau\rangle \leqslant Z(T)$, then (2.5) yields an elementary abelian group $\Pi$ containing $\sigma$, and an element $\varphi$ of $N(\Pi)$ of odd order moving $\sigma$. By (3.2), $\left\langle\sigma^{\delta} \mid \delta \in\langle\varphi\rangle\right\rangle$ must have order 4 , and once again (ii) follows.

(iii) Take $\Sigma$ as in (ii), and take a 3-element $\varphi \in N(\Sigma) \backslash C(\Sigma)$. Write $\Delta=\langle\varphi\rangle \Sigma O(\Gamma)$. By (4.1), $\Sigma$ centralizes $O(\Gamma)$. Thus all conjugates of $\sigma$ centralize $O(\Gamma)$.

Now $O(\Gamma)$ fixes the centre and axis of each involution in $\Sigma$. Suppose $O(\Gamma)$ fixes only these three points $x, y, z$. Then $\Gamma$ acts on $\{x, y, z\}$. Here $\Gamma \triangleright \Gamma_{x y \varepsilon}$, where $\Gamma_{x y z}$ centralizes $\Sigma$ by (3.1)(iii), and $\Gamma / \Gamma_{x y z}$ is $A_{3}$ or $S_{3}$. Thus, $\Gamma_{x y z} \geqslant O(\Gamma)$.

Next, suppose $O(\Gamma)$ fixes a point other than the centre of an involution in $\Sigma$. Then the transitivity of $N_{\Gamma}(\Sigma)$ on these centres shows that $O(\Gamma)$ is planar. Clearly $\Gamma$ acts on $\mathscr{P}_{O(\Gamma)}$, and each involutory homology $\rho$ of $\Gamma$ induces an involutory homology on $\mathscr{P}_{O(\Gamma)}$. In particular, the centre and axis of $\rho$ are in $\mathscr{P}_{O(\Gamma)}$. (3.1)(iii) shows that $\rho$ centralizes $\Gamma\left(\mathscr{P}_{O(\Gamma)}\right)$. Since $\Gamma^{*}$ is generated by all such homologies $\rho, \Gamma^{*}$ centralizes $\Gamma\left(\mathscr{P}_{O(\Gamma)}\right)$, so $O(\Gamma) \leqslant \Gamma\left(\mathscr{P}_{O(\Gamma)}\right) \leqslant C_{\Gamma}\left(\Gamma^{*}\right)$. This proves (iii) and (iv).

Proof of Theorem B. By (3.2)(ii) and (2.7), either (a) $\Gamma / O(\Gamma)$ is isomorphic to $\operatorname{PSL}(2, q), \operatorname{PGL}(2, q), \operatorname{PSL}(3, q), \operatorname{PSU}(3, q), \mathrm{A}_{7}, \operatorname{PSU}(3,4)$, or $\mathrm{M}_{11}$, where $q$ is odd, or (b) $\Gamma / O(\Gamma)$ has a normal subgroup $\left\langle\varphi_{1}\right\rangle \times\left\langle\varphi_{2}\right\rangle$, with $\left|\varphi_{1}\right|=\left|\varphi_{2}\right|=2^{e} \geqslant 2$, having quotient group $S_{3}$. By Theorem $A$, $O(\Gamma) \leqslant Z(\Gamma)$ in (a), while $O(\Gamma)$ centralizes the Klein group of $\left\langle\varphi_{1}\right\rangle \times\left\langle\varphi_{2}\right\rangle$ in (b).

It suffices to consider (a). By (2.8), we need to eliminate only the possibility $\Gamma \approx M_{11}$. Thus, assume $\Gamma$ is $M_{11}$, and regard it as represented as usual on a set $S$ of size 11. The stabilizer of an unordered pair from $S$ has a subgroup isomorphic to $S_{3} \times S_{3}$. By (3.6), we may assume that $\Gamma$ has a subgroup $\left\langle\sigma, \sigma^{\prime}\right\rangle \approx \mathrm{S}_{3}$ of perspectivities with centre $x$. Then $C(\sigma)<\Gamma_{x}$, so $\Gamma_{x}$ is precisely the stabilizer of an unordered pair from $S$. (Note that $\Gamma$ cannot fix $x$, as otherwise we would have $\Gamma \leqslant \Gamma(x)$.) Since 
$\sigma$ fixes exactly three elements of $S$, it fixes exactly $3+4$ pairs from $S$; moreover, $C(\sigma)$ has orbits of lengths 3 and 4 on these seven pairs.

Consequently $\sigma$ fixes exactly seven points in $x^{\Gamma}$, and $C(\sigma)$ permutes these points in orbits of lengths 3 and 4. Then $c_{\sigma} \notin x^{\Gamma}$, and the seven points are on $A_{\sigma}$. Let $\langle\sigma, \tau\rangle$ be a Klein group in $\Gamma$. As permutations of $S$, $\sigma$ and $\tau$ must interchange two fixed points of $\tau$ and $\sigma$, respectively. Thus, $\sigma$ and $\tau$ fix at least two common pairs. This means that $\left|A_{\sigma} \cap A_{\tau} \cap x^{\Gamma}\right| \geqslant 2$. Then $A_{\sigma}=A_{\tau}$, which is ridiculous.

\section{Proof of Theorem $\mathrm{C}$}

Let $\mathscr{P}, \Gamma$, and $n$ be as in Theorem C. Let $p$ be the prime dividing $q$.

Our approach will be to use the method described in [8, pp. 12-16]. More precisely, the points and lines of the desired subplane will be identified in terms of subgroups of $\Gamma$, with incidence defined by group containment.

Case 1. $\Gamma=\operatorname{PSL}(3, q)$ or $\operatorname{SL}(3, q)$. By considering the action of $\Gamma$ on $\operatorname{PG}(2, q)$, we can find a subgroup $\Delta_{1} \times \Delta_{2}$ with $\Delta_{1}$ and $\Delta_{2}$ dihedral groups of order $2 p$. By (3.6) and duality, we may assume that $\Delta_{1} \leqslant \Gamma(x)$ for some point $x$. If $\sigma \in \Delta_{1}$ is an involution, it follows that $C_{\Gamma}(\sigma)<\Gamma_{x}$. Clearly $\Gamma \neq \Gamma_{x}$ as $\Gamma \neq \Gamma(x)$. Thus $\left|\Gamma: \Gamma_{x}\right|=q^{2}+q+1$, and $\Gamma_{x}$ is the stabilizer of a point or line of $\mathrm{PG}(2, q)$.

Now $\sigma$ fixes $q+2$ points of $x^{\Gamma}$, which are permuted by $C(\sigma)$ in orbits of lengths 1 and $q+1$. Consequently $A_{\sigma} \cap x^{\Gamma}$ consists of $q+1$ points, which are also a line of $\mathrm{PG}(2, q)$. It is now clear that $x^{\Gamma}$, and the lines meeting it at least twice, form a subplane $\mathscr{P}_{0} \approx \mathrm{PG}(2, q)$.

Let $\Pi<\Gamma(L)$ induce a group of elations of $\mathscr{P}_{0}$ of order $q^{2}$. Then $\Pi$ is inverted in $\Gamma$. Thus, by (3.4), $q^{2} \mid n^{2}$ and hence $q \mid n$.

Let $\gamma \in \Gamma$ have order $q-1$, fix precisely three points of $\mathscr{P}_{0}$ (which are non-collinear), and be inverted in $\Gamma$. By (3.4), $(q-1) \mid(n-1)$.

Let $\delta \in \Gamma$ have order $q+1$, fix precisely one line $L$ of $\mathscr{P}_{0}$ and one point $x \notin L$ of $\mathscr{P}_{0}$, and be inverted in $\Gamma$. By (3.4) (applied to each prime power divisor of $q+1),(q+1) \mid\left(n^{2}-1\right)$.

Case 2. $\Gamma=\operatorname{PGL}(2, q)$ with $(q, n) \neq 1$. Let $\Pi$ be a Sylow $p$-subgroup of $\Gamma$. Then $\Pi$ has order $q$, and is inverted by an involution. We can apply (3.5). As $p \mid n$ and $N_{\Gamma}(\Pi)$ does not have $S_{3}$ as a homomorphic image, $N_{\Gamma}(\Pi)$ fixes a point $x$ and line $X$. By (3.4), $x \in X$ since $p \mid n$. Clearly $x^{\Gamma}$ has $q+1$ points, and $\Gamma$ acts 3 -transitively on $x^{\Gamma}$.

Let $\mathscr{C}$ and $\mathscr{A}$ be the sets of centres and axes of involutions in $\Gamma$. We shall show that $x^{\Gamma} \cup \mathscr{C}$ and $X^{\Gamma} \cup \mathscr{A}$ are the points and lines of a desarguesian subplane of order $q$. Clearly each of these sets has $q+1+q^{2}$ elements. 
If $c_{\sigma}, c_{\tau} \in \mathscr{C}$ with $\sigma$ and $\tau$ different involutions satisfying $|\sigma \tau| \neq p$, the structure of $\Gamma$ ensures the existence of a unique involution $\rho \neq \sigma, \tau$ centralizing $\langle\sigma, \tau\rangle$, and then $c_{\sigma}, c_{\tau} \in A_{\rho}$. Similarly, if $\sigma \tau \in \Pi$, then $\sigma, \tau \in N_{\Gamma}(\Pi)=\Gamma_{X}$, so $c_{\sigma}, c_{\tau} \in X\left(\right.$ since $\left.A_{\sigma} \neq X\right)$.

Consider $x$ and $c_{\sigma}$. If $\sigma$ normalizes $\Pi$, then $c_{\sigma} \in X$. Suppose $\sigma \notin N_{\Gamma}(\Pi)=\Gamma_{x}$. Then $\sigma$ centralizes the unique involution $\tau$ in $\Gamma_{x x^{\sigma}}$, so $c_{\sigma} \in A_{\tau}$. Also, $\tau \in N_{\Gamma}(\Pi)=\Gamma_{x}$, so $x \in A_{\tau}$ (since $x \neq c_{\tau}$ ).

The preceding observations, and their duals, show that all incidences between $x^{\Gamma} \cup \mathscr{C}$ and $X^{\Gamma} \cup \mathscr{A}$ are the same as in a desarguesian plane of order $q$. We have thus constructed the desired subplane $\mathscr{P}_{0}$ of $\mathscr{P}$.

As in Case $1,(q-1) \mid(n-1)$ and $(q+1) \mid\left(n^{2}-1\right)$. $\Pi$ contains no nontrivial elations of $\mathscr{P}_{0}$. Hence, by (3.4), $\Pi$ is semiregular off $X$, so $q \mid n^{2}$.

Case 3. $\Gamma=\operatorname{PSU}(3, q)$ or $\operatorname{SU}(3, q)$ with $(q, n) \neq 1$. Let $\Pi$ be a Sylow $p$-subgroup of $\Gamma$, so $|\Pi|=q^{3}$. Since $p \mid n$, $\Pi$ fixes a line $X$. If $\Pi$ fixes $X^{\prime} \neq X$, it fixes $x=X \cap X^{\prime}$, and hence (since $p \nmid(n-1)$ ) a third line on $x$. Each element of $\Pi \backslash Z(\Pi)$ is inverted by an involution, so by (3.4) each fixed line of $\Pi$ passes through $x$. Thus $N_{\Gamma}(\Pi)$ fixes $x$, so that $\left|x^{\Gamma}\right|=q^{3}+1$ and $\Gamma$ is 2-transitive on $x^{\Gamma}$.

Similarly, if $\Pi$ fixes just one line $X$, then $N_{\Gamma}(\Pi) \leqslant \Gamma_{X}$. Dualizing, and proceeding as before, we again find that there is a point $x$ such that $\left|x^{\Gamma}\right|=q^{3}+1$ and $\Gamma$ is 2 -transitive on $x^{\Gamma}$.

For any involution $\sigma, C_{\Gamma}(\sigma)$ is transitive on the fixed points of $\sigma$ in $x^{\Gamma}$, so $A_{\sigma} \cap x^{\Gamma}$ is a line of the usual unital defined on $x^{\Gamma}$. Consequently, if a line of $\mathscr{P}$ contains two points of $x^{\Gamma}$ it contains exactly $q+1$ points.

Let $\sigma$ and $\tau$ be any distinct involutions in $\Gamma_{x}$. Then $|\sigma \tau|=p \mid n$. By (3.4), $x=A_{\sigma} \cap A_{\tau} \in c_{\sigma} c_{\tau}$, so $c_{\tau} \in x c_{\sigma}$. Consequently, all centres of involutions in $\Gamma_{x}$ lie on a line, which is clearly fixed by $\Pi$, and hence may be assumed to be $X$. In fact, even $N_{\Gamma}(\Pi)=\Gamma_{x}$ must fix $X$.

Now, precisely as in Case 2, we can use $x^{\Gamma}, X^{\Gamma}$, and the centres and axes of involutions of $\Gamma$, to construct a desarguesian subplane $\mathscr{P}_{0}$ of order $q^{2}$. (The existence of a unique involution commuting with two given involutions $\sigma, \tau$ with $p \nmid|\sigma \tau|$ is evident from the case in which $\mathscr{P}=\mathrm{PG}\left(2, q^{2}\right)$.)

$\Pi$ acts on the $n^{2}$ points off $X$. Since each element of $\Pi \backslash Z(\Pi)$ is inverted by an involution and does not induce an elation of $\mathscr{P}_{0},(3.4)$ shows that the stabilizer in $\Pi$ of a point off $X$ must be in $Z(\Pi)$. Thus, $q^{2} \mid n^{2}$. The remainder of Case 3 is proved as in Case 1.

\section{2-groups}

In this section we shall obtain some additional information concerning the situation in Theorem A. 
(7.1) Lemma. Let $\mathscr{P}$ be a projective plane of odd order, and $T$ a 2-group of collineations of $\mathscr{P}$ containing commuting involutory homologies having different axes. Let $\tau \in T \backslash Z(T)$ be an involutory homology which is a square in $T$. Then there is an involutory homology $\sigma \in Z(T)$ such that $\langle\sigma, \tau\rangle \triangleleft T$.

Proof. By (3.3), $Z(T)$ contains an involutory homology $\sigma$. Suppose $\langle\sigma, \tau\rangle$ is not normal in $T$, and let $\tau=\gamma^{2}$ with $\gamma \in T$. Then $\gamma \in N_{T}(\langle\sigma, \tau\rangle)$. Let $\varphi \in T \backslash N_{T}(\langle\sigma, \tau\rangle)$ normalize $N_{T}(\langle\sigma, \tau\rangle)$. Then $\tau^{\varphi}$ and $\gamma^{\varphi}$ are in $N_{T}(\langle\sigma, \tau\rangle) \backslash\langle\sigma, \tau\rangle$. Since $\gamma^{\phi}$ centralizes $\sigma$ and acts on $\langle\sigma, \tau\rangle, \tau^{\varphi}=\left(\gamma^{\varphi}\right)^{2}$ centralizes $\langle\sigma, \tau\rangle$. This contradicts (3.2).

In the remainder of this section, $\mathscr{P}, \Gamma$, and $\sigma$ will be as in Theorem A. Let $T$ be a Sylow 2-subgroup of $\Gamma$ such that $\sigma \in Z(T)$ and $\Sigma \leqslant T$. Write $\Gamma^{+}=\left\langle\sigma^{\Gamma}\right\rangle$.

(7.2) Lemma. If $T$ has no normal Klein subgroup, then $\Gamma$ has no Baer involutions (so Theorem B applies).

Proof. By [12, p. 199], $T$ must be dihedral or quasidihedral. Suppose $T$ is dihedral. By Theorem A(ii), $T \backslash\langle\sigma\rangle$ contains a conjugate of $\sigma$. If $T$ also contains a Baer involution $\alpha, T$ has a dihedral subgroup $T_{0}$ of index 2 containing no Baer involution. Clearly $T \backslash T_{0}$ cannot contain a homology. By (2.6), $\Gamma$ has a normal subgroup $\Gamma_{0}$ of index 2 such that $\Gamma_{0} \cap T=T_{0}$. Then $\Gamma \backslash \Gamma_{0}$ contains no involutory homology, whereas $\Gamma$ is generated by $O(\Gamma)$ and by its involutory homologies.

If $T$ is quasidihedral, it has just one class of involutions other than $\sigma$, so the lemma is clear by Theorem A(ii).

(7.3) Lemma. If $\left\langle\sigma^{\Gamma} \cap T\right\rangle$ is a Klein group, then $\Gamma$ has no Baer involutions (so Theorem $B$ applies).

Proof. By Theorem A(ii), all involutions in $\Sigma=\left\langle\sigma^{\Gamma} \cap T\right\rangle$ are conjugate. Consequently $\Sigma^{\gamma} \cap T \leqslant \Sigma$ for all $\gamma \in \Gamma$. Now Goldschmidt's theorem on strongly closed abelian subgroups [11] shows that $\sigma^{\Gamma}$ is the set of all involutions in $\Gamma^{+}=\left\langle\sigma^{\Gamma}\right\rangle$, so Theorem B applies to $\Gamma^{+}$.

If Theorem $\mathrm{B}(\mathrm{a})$ holds, then $\Gamma^{+}$is $\operatorname{PSL}(2, q)(q \equiv 3$ or $5 \bmod 8)$, $\operatorname{PSU}(3,4), A_{7}$, or $\hat{A}_{7}$. We may assume $\Gamma^{*}>\Gamma^{+}$, so $\Gamma^{*} \backslash \Gamma^{+}$contains an involutory homology $\rho$. Using (3.2), we find that $\Gamma^{*}$ is $\operatorname{PGL}(2, q)$, so all involutions in $\Gamma^{*}$ (and hence in $\Gamma=O(\Gamma) \Gamma^{*}$ ) are conjugate to $\sigma$ or $\rho$. If Theorem $B(b)$ holds for $\Gamma^{+}$, the same argument shows that $\Gamma^{*}=\Gamma^{+}$.

(7.4) Propositron. If $T$ has no normal Klein subgroup generated by involutory homologies, then $\left\langle\sigma^{\mathrm{\Gamma}} \cap T\right\rangle$ is dihedral. 
Proof. We first claim that if $\tau \in T \backslash\langle\sigma\rangle$ is conjugate in $\Gamma$ to $\sigma$, then $C_{T}(\tau)$ has no element with square $\sigma$. For, suppose $C_{T}(\tau)$ has such an element. By applying Sylow's theorem to $C_{\Gamma}(\sigma)$, we can find $\gamma \in \Gamma$ such that $\tau^{\gamma}=\sigma$ and $C_{T}(\tau)^{\gamma} \leqslant T$. Now $C_{T}(\tau)^{\gamma}$ has an element whose square is $\sigma^{\gamma} \neq \tau^{\gamma}$, and this contradicts (7.1).

Let $\Delta$ be a dihedral subgroup of $T$ maximal with respect to being generated by two elements of $\sigma^{\Gamma}$. There can be no $\tau \in \sigma^{\Gamma} \cap(T \backslash \Delta)$ normalizing $\Delta$. For, if there is such a $\tau$, consider $\Delta\langle\tau\rangle$. By (3.2) and the preceding paragraph, $C_{\Delta}(\tau)=\langle\sigma\rangle$. Then $\Delta\langle\tau\rangle$ must be dihedral, and this contradicts the maximality of $\Delta$.

We can now show that $\Delta \unlhd T$. For, if $N_{T}(\Delta)<T$, let $\varphi \in T \backslash N_{T}(\Delta)$ normalize $N_{T}(\Delta)$. Then $\Delta^{\varphi}$ normalizes $\Delta$, while $\Delta^{\varphi} \neq \Delta$, and this contradicts the preceding paragraph.

Finally, the normality of $\Delta$, together with its maximality, imply that $\Delta$ contains $\sigma^{\Gamma} \cap T$. This completes the proof.

A more detailed version of the preceding argument yields information even if $T$ has a normal Klein subgroup generated by involutory homologies. However, we have not been able to determine $\left\langle\sigma^{\Gamma} \cap T\right\rangle$ in this case (it should be dihedral of order 8 , or be generated by three involutory homologies $\mu, \nu, \tau$, such that $\mu^{\tau}=\sigma \mu$ and $\nu^{\tau}=\sigma \nu$, where $\sigma \in\langle\mu \nu\rangle$ is an involutory homology).

\section{Concluding remarks}

Let $\mathscr{P}$ be a projective plane of order $n$, and $\Gamma$ a collineation group of even order generated by involutory perspectivities.

RemarK 1. Suppose $n$ is even and $\Gamma$ contains involutory elations with different centres or axes. Then, using [25, Theorem 3, and 14], it is easy to show that the following are the only possibilities for $\Gamma: \Gamma$ is isomorphic to $\operatorname{PSL}\left(2,2^{e}\right), \operatorname{PSL}\left(3,2^{e}\right)$, SL $\left(3,2^{e}\right), \operatorname{PSU}\left(3,2^{e}\right), \operatorname{SU}\left(3,2^{e}\right)$, or $\mathrm{Sz}\left(2^{e}\right) ; \Gamma$ is a 2 -group; $|\Gamma| \equiv 2 \bmod 4$; or $\Gamma$ has a normal elementary abelian subgroup $\Delta$, consisting of elations having the same centre or axis, and $\Gamma / \Delta$ is isomorphic to $\operatorname{PSL}\left(2,2^{e}\right), \operatorname{PSU}\left(3,2^{e}\right)$, or $\operatorname{Sz}\left(2^{e}\right)$, or $|\Gamma / \Delta| \equiv 2$ $\bmod 4$.

It is also easy to show that, if $\Gamma \approx \operatorname{PSL}\left(2,2^{e}\right), \operatorname{PSL}\left(3,2^{e}\right)$, or $\operatorname{SL}\left(3,2^{e}\right)$, then $\mathscr{P}$ has a desarguesian $\Gamma$-invariant subplane of order $2^{e}$. Moreover, $2^{e}\left|n,\left(2^{e}-1\right)\right|(n-1)$, and $\left(2^{e}+1\right) \mid\left(n^{2}-1\right)$.

However, as pointed out to me by Hering, the cases $\Gamma \approx \operatorname{PSU}\left(3,2^{e}\right)$ or $\mathrm{SU}\left(3,2^{e}\right)$ are difficult to handle. It is easy to see that (by duality) it may be assumed that $\Gamma$ has a point-orbit $x^{\Gamma}$ of size $2^{3 e}+1$. If $x^{\Gamma}$ is contained in 
a line, nothing is known. If $x^{\Gamma}$ is not contained in a line, there should be a line-orbit of size $2^{3 e}+1$, but this seems very difficult to prove. This is one of the rare instances where even order planes present difficulties not found for odd order ones. Namely, if $L$ is a line and $\left|L \cap x^{\Gamma}\right| \geqslant 2$, then $\left|L \cap x^{\Gamma}\right|=2^{e}+1$. It is now conceivable that every element of $\Gamma\left(L \cap x^{\Gamma}\right)$ (which is a group of order $q+1$ or $\frac{1}{3}(q+1)$ ) is planar, a situation that did not arise in Theorem C. On the other hand, if some such element is not planar, the desired desarguesian subplane of order $2^{2 e}$ can, indeed, be constructed.

REMARK 2. Suppose $n$ is odd, and suppose $\Gamma$ contains a Klein group with centre $x$, but $\Gamma \neq \Gamma(x)$. This is a situation specifically excluded in Theorems A, B, and C. In this case, $\Gamma$ can be easily determined using [14] (see [15] and [17, (2.5)]): $\Gamma \approx \mathrm{SL}\left(2,2^{e}\right), \operatorname{PSU}\left(3,2^{e}\right), \mathrm{SU}\left(3,2^{e}\right)$, or $\mathrm{Sz}\left(2^{e}\right)$. However, it seems unlikely that any of these possibilities occurs.

By (2.3), if $\Gamma$ contains an involutory homology but no Klein group consisting of homologies, then $|\Gamma| \equiv 2 \bmod 4$.

Remark 3. Suppose $\Gamma$ is as in Theorem $B$, and $\Gamma$ is isomorphic to $\operatorname{PSL}(2, q)(q \equiv 1 \bmod 4), \operatorname{PGL}(2, q), \operatorname{PSU}(3, q)$, or $\mathrm{SU}(3, q)$. Assume that $(q, 3 n)=1$. Then $\Gamma$ has point- and line-orbits of size $q+1$ or $q^{3}+1$. Moreover, the incidence structure of centres and axes of involutory perspectivities is isomorphic to that of $\mathrm{PG}(2, q)$ or $\mathrm{PG}\left(2, q^{2}\right)$. Also, (3.4) provides some additional numerical information, but the impossibility of this situation remains to be proved.

We note that PSL $(3,25)$ has a subgroup isomorphic to $\operatorname{PSL}(2,9)$. This seems to make the case $q=3^{e}$ particularly difficult.

Remark 4. Case (b) of Theorems A(iii) and B actually occurs. (For example, take $\mathrm{PG}(2, q), q \equiv 1 \bmod 4$, and let $\Gamma$ be the stabilizer of a triangle in $\operatorname{PSL}(3, q)$ or $\operatorname{PrL}(3, q)$.) Note that, in Theorem $\mathrm{B}(\mathrm{b})$, a Sylow 2-subgroup of $\Gamma$ is wreathed: $Z_{2^{\mathrm{e}}} \operatorname{wr} Z_{2}, e \geqslant 2$.

REMARK 5. We have already mentioned several times that many of our results hold for finite collineation groups of infinite planes, provided of course, that numerical information pertaining to the order of the plane is deleted. Thus, all results of $\S \S 3,4,5$, and 7 , together with Theorem $\mathrm{C}(\mathrm{i})$, have versions holding in the infinite case.

Remark 6. The arguments used in (3.5), (4.1), and Theorem A(iii) do not use the prime 2 in a significant way. Thus corresponding results can be proved concerning central collineations of other orders. However, such 
general results would be difficult to apply to the problem of classifying finite collineation groups, in view of the central role of involutions in this problem.

\section{REFERENCES}

1. J. Alperin, R. Brauer, and D. Gorenstenn, 'Finite simple groups of 2-rank two', Scripta Math. 29 (1973) 191-214.

2. H. BENDER, 'Transitive Gruppen gerader Ordnung, in denen jede Involution genau einen Punkt festlasst', J. Algebra 17 (1971) 527-54.

3. R. Brauer, 'Some application of the theory of blocks of characters of finite groups II', ibid. I (1964) 307-34.

4. H. Burgoyne and P. Fong, 'Multipliers of the Mathieu groups', Nagoya Math J. 27 (1966) 733-45.

5. W. BURnside, Theory of groups of finite order, 2nd edn (Dover, New York, 1955).

6. P. Demвowskx, 'Gruppentheoretische Kennzeichnungen der endlichen desarguesschen Ebenen', Abh. Math. Sem. Univ. Hamburg 29 (1965) 92-106.

7. - 'Zur Geometrie der Suzukigruppen', Math. Z. 94 (1966) 106-09.

8. - Finite geometries (Springer, Berlin, 1968).

9. W. Feit and J. G. Thompson, 'Solvability of groups of odd order', Pacific J. Math. 13 (1963) 771-1029.

10. G. Glauberman, 'Central elements in core-free groups', J. Algebra 4 (1966) 403-20.

11. D. Goldschmidx, '2-Fusion in finite groups', Ann. of Math. 99 (1974) 70-117.

12. D. GoReNstenN, Finite groups (Harper, New York, 1968).

13. R. Griess, 'Schur multipliers of the known finite simple groups', Bull. Amer. Math. Soc. 78 (1972) 68-71.

14. C. Hering, 'On subgroups with trivial normalizer intersection', J. Algebra 20 (1972) 622-29.

15. _Eine Bemerkung über Streckungsgruppen', Arch. Math. (Basel) 23 (1972) 348-50.

16. A. R. Hoffer, 'On unitary collineation groups', J. Algebra 22 (1972) 211-18.

17. W. M. Kantor, 'On unitary polarities of finite projective planes', Canad. $J$. Math. 23 (1971) 1060-77.

18. - 'On homologies of finite projective planes', Israel J. Math. 16 (1973) 351-61.

19. - 'Those nasty Baer involutions', Proc. Int. Conf. Proj. Planes (Washington State University Press, 1973), pp. 145-55.

20. H. LÜNEBURG, 'Zur Frage der Existenz von endlichen projektiven Ebenen vom Lenz-Barlotti-Typ III-2', J. reine angew. Math. 220 (1965) 63-67.

21. - 'Charakterisierungen der endlichen desarguesschen projektiven Ebenen', Math. Z. 85 (1964) 419-50.

22. 'Uber projective Ebenen, in denen jede Fahne von einer nichttrivialen Elation invariant gelassen wird', Abh. Math. Sem. Univ. Hamburg 29 (1965) $37-76$.

23. T. G. Ostrom, 'Double transitivity in finite projective planes', Canad. J. Math. 8 (1956) 563-67.

24. - 'A characterization of the Hughes planes', ibid. 17 (1965) 916-22.

25. F. C. PIPER, 'Collineation groups containing homologies', J. Algebra 6 (1967) 256-69.

26. I. SchUR, 'Untersuchungen über die Darstellungen der endlichen Gruppen durch gebrochene lineare Substitutionen', J. reine angew. Math. 132 (1907) 85-137. 
27. H. Unkelbach, 'Eine Charakterisierungen der endlichen Hughes-Ebenen', Geometriae Dedicata 1 (1973) 148-59.

28. J. Y YQUB, 'On two theorems of Lüneburg', Arch. Math. (Basel) 17 (1966) 485-88.

University of Oregon

Eugene, Oregon 97403 